Historias de Vida de estudiantes universitarios migrantes. Páginas 249-268 en Revista de la Escuela de Ciencias DE LA EDUCACIÓN, AÑo 11, NÚMERO 10, ENERO A DICIEMBRE DE 2015. ISSN 1851-6297. ISSN EN LÍNEA 2362-3349.

\title{
HISTORIAS DE VIDA DE ESTUDIANTES UNIVERSITARIOS MIGRANTES
}

\author{
Por Guadalupe R. Leiva* \\ Universidad Nacional del Nordeste - CONICET, Argentina. \\ guadalupeleiva11@gmail.com
}

Recibido: 30/06/2014 Aceptado: 27/08/2014

\section{Resumen}

El artículo científico corresponde a los avances de la investigación titulada "Movimientos migratorios en torno a la formación: dos historias de vida" (1) realizada en el marco de la beca de perfeccionamiento (2013-2015) de la Secretaría General de Ciencia y Técnica de la Universidad Nacional del Nordeste (UNNE).

La problemática de estudio resulta pertinente y relevante dado que la UNNE responde a la demanda de formación de grado de las provincias que representan a la región NEA de la República Argentina.

El propósito sustantivo ha sido descubrir el modo en el que ciertos acontecimientos propios de los movimientos migratorios de los recién graduados de una carrera universitaria impactaron en el trayecto de formación, intentando identificar el significado que le otorgan a dichos acontecimientos.

La perspectiva metodológica de la investigación se sustenta en una lógica cualitativa y recursiva, se trabajó sobre el análisis de casos.

\section{Palabras clave:}

Migración - Acontecimiento - Formación - Universidad - Estudiantes.

\section{Abstract}

The scientific paper corresponds to the progress of research entitled "Migratory movements around training: two stories of life" (2), held in the framework of the

* Prof. y Lic. en Ciencias de la Educación (Facultad de Humanidades de la Universidad Nacional del Nordeste). Doctoranda en Educación (Universidad Nacional de Entre Ríos). Becaria doctoral UNNE - CONICET. Profesora auxiliar 1ra categoría simple de las cátedras "Grupos e Instituciones educativas" y "Psicosociología de la Formación" (Ciencias de la Educación - Facultad de Humanidades de la UNNE. 
Revista de la Escuela de Ciencias de la Educación, año 11, número 10, enero a diciembre de 2015. Páginas 249-268. ISSN 1851-6297. ISSN EN LINEA 2362-3349. HISTORIAS DE VIDA DE ESTUDIANTES UNIVERSITARIOS MIGRANTES. GUADALUPE R. LEIVA

development grant (2013 - 2015) from the General Secretariat of Science and Technology, National University of the Northeast (in Spanish UNNE).

The problem of study is pertinent and relevant as the UNNE responds to the demand for undergraduate education in the provinces representing the region of northeastern Argentina.

The substantive purpose was to discover the way in which certain own migration events of recent graduates of a university career hit the training path, trying to identify the significance given to these events.

The methodological approach of the research is based on a qualitative and recursive logic, we worked on the case analysis.

\section{Keywords:}

Migration - Event - Training - University - Students.

\section{Introducción}

El presente artículo científico tiene como objetivo comunicar los avances de la investigación correspondiente a la beca de perfeccionamiento (20132015) titulada "Movimientos migratorios en torno a la formación: dos historias de vida" (3).

Uno de los antecedentes es la investigación que desarrollé en el marco de la beca de iniciación (Secretaría General de Ciencia y Técnica de la Universidad Nacional del Nordeste) titulada "Las representaciones que tienen los alumnos de Ciencias de la Educación sobre la formación y los espacios de acompañamiento" realizada durante los años 2010-2013. El sentido de identidad y pertenencia al grupo de pares en la universidad fue una de las invariantes del caso; los estudiantes, en sus argumentaciones sobre las representaciones que tienen acerca del acompañamiento -basándose en la experiencia cotidiana estudiantil- remarcaron en reiteradas oportunidades la figura del par; destacando ciertos aspectos relativos a: las tareas propias del momento del cursado de las materias, las características singulares de cada uno de los grupos de estudio, la seguridad que le brinda la presencia del par en su trayecto académico y los movimientos migratorios realizados durante su trayecto de formación universitaria. Una de las líneas de análisis que se ha desprendido de ese estudio, hace referencia a la figura del compañero como aquel que acompaña su recorrido académico, operando como apoyatura (4) en determinados momentos. De este modo, el grupo de pares -en tanto apoyatura- posibilita al alumno que migra la reconfiguración de su identidad.

A partir de los resultados de esta investigación se pudo advertir la importancia de los movimientos migratorios en sujetos en formación, y desde allí se formula la pregunta problema: ¿Cuál es el impacto que tienen para el sujeto ciertos acontecimientos propios de los movimientos migratorios en su trayecto de formación? 
Revista de la Escuela de Ciencias de la Educación, año 11, número 10, enero a diciembre de 2015. Páginas 249-268. ISSN 1851-6297. ISSN EN LINEA 2362-3349. HISTORIAS DE VIDA DE ESTUDIANTES UNIVERSITARIOS MIGRANTES. GUADALUPE R. LEIVA

Los objetivos generales de conocimiento de esta investigación son descubrir el modo en que ciertos acontecimientos propios de los movimientos migratorios inciden en el trayecto de formación y relacionar los resultados de esta investigación con la información proveniente de la anteriormente trabajada (5).

El objetivo de intervención es formular líneas de interpretación sobre los modos de abordar el proceso de formación y acompañamiento que atiendan a los posibles impactos (6) de los movimientos migratorios de tipo geográficos del sujeto en formación.

Los objetivos específicos son identificar los acontecimientos que el sujeto recupera como significativos en el relato de su historia de formación, para luego descubrir el significado que le otorga a dichos acontecimientos y a los movimientos migratorios recuperados en el relato de su historia de vida.

El supuesto de partida de esta investigación se basa en la idea de que los movimientos migratorios rompen con las configuraciones vinculares de los sujetos; por lo cual, la migración es vivida como ruptura, separación y desarraigo. Es la capacidad de transformar tales situaciones críticas en experiencias de aprendizaje a partir de interacciones que construyen con el medio y con "los otros" individuo así se posibilita la estabilidad de su self y su sentimiento de identidad.

\section{Encuadre referencial de la investigación}

Las nociones sustantivas para el abordaje de esta investigación fueron: migración, acontecimiento, formación e historias de vida. A continuación se presenta una breve referencia sobre cada una de ellas para dar cuenta de la relación intrínseca que guardan con el objeto de estudio planteado.

Este trabajo adhiriere a la segunda acepción de la noción de migración (Del lat. migratǐo, -ōnis) tal como es expresado en el Diccionario de la Lengua Española (2009)

Acción y efecto de pasar de un país a otro para establecerse en él. Se usa hablando de las migraciones históricas que hicieron las razas o los pueblos enteros. // Desplazamiento geográfico de individuos o grupos, generalmente por causas económicas o sociales.

La noción de movimiento entendido así, hace referencia a la acción y efecto del estado del cuerpo de los sujetos en relación con los cambios de posición o de lugar geográfico (movimientos migratorios) que puede o no provocar algún impacto en los sujetos.

Lo migratorio entonces, se convertiría en el adjetivo que refiere a aquel que migra o realiza algún tipo de desplazamiento y que pone al sujeto a prueba, en relación con sus configuraciones vinculares frente a lo imprevisto, lo incierto, lo ajeno del otro y la capacidad de transformar las situaciones críticas en experiencias de aprendizaje - parafraseando a Grinberg y Grinberg (1976, p.139). 
Revista de la Escuela de Ciencias de la Educación, año 11, número 10, enero a diciembre de 2015. Páginas 249-268. ISSN 1851-6297. ISSN EN LINEA 2362-3349. HISTORIAS DE VIDA DE ESTUDIANTES UNIVERSITARIOS MIGRANTES. GUADALUPE R. LEIVA

En la medida en que el individuo puede ir elaborando tal experiencia a lo largo del tiempo, va integrando los aspectos y sentimientos negados y disociados para poder padecer su dolor.

En tal sentido, los sujetos migrantes se ven interpelados por la nueva realidad que demanda la modificación de sus estructuras internas; este proceso es vivenciado como momento de dolor, desorganización, frustración y puede constituirse en un cambio catastrófico (7).

Podría pensarse la migración como "lo siniestro" término utilizado por Freud (2006, p. 2483) en relación con el lugar, donde éste se vuelve extraño y por desconocido se lo teme. Sin bien, lo nuevo no necesariamente es para el sujeto algo "siniestro", para que adquiera tal característica debe estar acompañado por algo más que aquello que sólo resulta desconocido. En tanto, la experiencia de migración conmueve al sujeto, generando incertidumbre, inseguridad, desconfianza y el sujeto siente amenazados sus referentes identitarios, su noción de tiempo, de espacio y su mismidad.

Al pensar en la experiencia cultural como el encuadre del sujeto -en términos de Kaës (1997, p. 30)-, la migración representa la discontinuidad de sus referentes, que se vivencia como ataque a la integridad del yo, es por ello que los sujetos que migran requieren de un espacio potencial que le sirva de lugar de transición, permitiéndole organizar sus partes del yo disueltas.

Los momentos de crisis son superados y elaborados, mediante "la transicionalidad de una experiencia de ruptura en la continuidad" (Winnicott, 1962 citado en Kaës, 1997, p. 61). La transicionalidad entonces puede entenderse como el proceso que corresponde o no al restablecimiento de las funciones fundamentales interrumpidas o modificadas frente a la migración; mediante la cual, los sujetos tendrán la posibilidad de restablecer la capacidad de simbolización y de creación.

La noción de acontecimiento, es aquí entendida desde los aportes de la hermenéutica de Ricoeur (2013, p. 13), como la actualización de los sentidos; el acontecimiento es la experiencia entendida como expresión.

Reconocer los acontecimientos narrados por los sujetos, nos llevaría a pensar en la tarea del traductor como la de traducir lo intraducible tal como lo plantea Ricoeur (2005, pp.61-75), como el acto de traducir lo étranger (Berman, 1995 citado por Ricoeur, 2005, p. 18) -termino que abarca la obra, el autor, su lengua $-\mathrm{y}$ el lector destinatario, donde las historias de vida se transforman en el lugar de acogida. Mediante la narración es posible descubrir el impacto de los movimientos migratorios en el trayecto de formación, donde habita el afán por por comprender en el sentido de acontecimiento y de significado atribuido por el sujeto.

La noción de formación, está ligada a la idea de dar una forma, de una especie de molde, cuya etimología se encuentra en la palabra italiana formaggio, dar forma a un trayecto personal y profesional según Ardoino (2005, p. 
Revista de la Escuela de Ciencias de la Educación, año 11, número 10, enero a diciembre de 2015. Páginas 249-268. ISSN 1851-6297. ISSN EN LINEA 2362-3349. HISTORIAS DE VIDA DE ESTUDIANTES UNIVERSITARIOS MIGRANTES. GUADALUPE R. LEIVA

25). En esta línea, Ferry (1997, pp. 53-54) plantea la idea de un sujeto que se forma a si mismo pero siempre por la mediación de formadores, de situaciones accidentales, sucesos críticos o bien, desde algún dispositivo. La formación es entendida, no sólo como la capacidad de adquirir conocimientos y de educarse, sino también como un cambio de actitud, de construcción y elaboración constante que les permitiría encontrarse consigo mismo en torno a su desarrollo personal.

En la reconstrucción de las historias de vida cada sujeto, en el acto de recordar, atribuye un orden, coherencia y un significado al propio trayecto de formación y de ahí su importancia como dispositivo de formación. De esta manera la identidad se convierte en narrativa mediante el acto del habla, es así como van recordando los hechos, relatándolos y reconstruyendo su propia historia de vida. El tiempo vivido y de los acontecimientos significativos para cada uno de los sujetos, adquiere entonces vital importancia.

Esta reconstrucción de la historia de vida de los individuos, se organiza en torno del núcleo de memoria (8) (Bachelard, 1950 citado por Candau, 2006, p. 102), entendido como el núcleo de sentido con el que los sujetos recuperan los acontecimientos significativos mediante el acto de la narración.

\section{Encuadre metodológico de la investigación}

La perspectiva metodológica de la investigación se sustenta en una lógica cualitativa y recursiva, mediante la cual, el hecho social se construye a partir de las historias de vida. Se trabajó sobre el análisis de casos (9) seleccionados por un interés "intrínseco" tal como lo entiende Stake (1999, p. 16) que proviene de los estudios realizados (10) en el marco de beca de iniciación en investigación obtenida con anterioridad.

El universo de investigación comprende a los graduados la carrera de profesorado en Ciencias de la Educación de una universidad nacional, que han participado en la investigación: "Las representaciones que tienen los alumnos de Ciencias de la Educación sobre la formación y los espacios de acompañamiento". La unidad de análisis está compuesta por dos recientes graduados, que participaron de las entrevistas que formaron parte del trabajo de investigación recién mencionado y que atravesaron uno o varios movimientos migratorios en su trayecto de formación.

La fuente de información fue la entrevista abierta en profundidad (11) sobre historias de vida (12) (HdV). Se realizaron tres ciclos de entrevistas, cada una con una devolución intermedia en la que se le facilitó al entrevistado una copia con la desgrabación de lo registrado en cada encuentro. Y para finalizar, se concluyó en un último encuentro con cada uno de los entrevistados, en el que se compartió el material recolectado y reconstruido por el investigador a través de distintos dispositivos de objetivación. 
Revista de la Escuela de Ciencias de la Educación, año 11, número 10, enero a diciembre de 2015. Páginas 249-268. ISSN 1851-6297. ISSN EN LINEA 2362-3349. HISTORIAS DE VIDA DE ESTUDIANTES UNIVERSITARIOS MIGRANTES. GUADALUPE R. LEIVA

En síntesis, el trabajo se sustentó en sucesivos acercamientos al objeto de estudio mediante:

a) entrevistas en profundidad en tres ciclos;

b) reconstrucción de la información recolectada con los diferentes dispositivos de objetivación de la información: recuperación cronológica de los hechos narrados, mapas de desplazamientos geográficos, elaboración del árbol genealógico y líneas de tiempo de la historia de cada sujeto en la que se ubicaron los acontecimientos cruciales;

c) elaboración de las primeras hipótesis interpretativas sobre el significado que han tenido los hechos relatados y las relaciones posibles entre ese significado y las características del relato.

En simultáneo se revisaron las perspectivas y aportes del marco conceptual, éstos operaron como un tercero que mediatizaron en la primera interpretación intentando dar respuestas a las preguntas iniciales.

\section{Reconstrucción de las historias de vida}

A partir del primer acercamiento al material recolectado en las entrevistas en profundidad con cada uno de los recientes graduados, se ha realizado la reconstrucción de cada una de las historias de vida, las que se describen a continuación:

\section{- Caso 1: Historia de vida de Daniel (13)}

El 18 de septiembre de 1987, en la ciudad de Montecarlo (Misiones) nace Daniel, siendo el segundo hijo del matrimonio de Margarita y Héctor. Sus primeros años, su infancia, su niñez y su adolescencia transcurren en la localidad mencionada. Su madre se desempeñaba como maestra en una de las escuelas de educación primaria del lugar; pertenecía a una familia en la que seis de los siete hermanos ejercían la docencia. Su padre era gendarme nacional y trabajaba en la base militar asentada en Montecarlo. Daniel recuerda que los días de su niñez estaban llenos de tareas extra escolares por parte de su madre.

La familia se reunía los días domingos, venían sus tíos y sus primos y en esas ocasiones, las charlas estaban relacionadas con la educación y las tareas sobre la profesión daban cita en su casa. Durante estos encuentros familiares, los chicos eran los encargados de pintar, recortar y ayudar a sus padres en las construcciones de los materiales que trabajarían luego en sus aulas.

De su infancia, recuerda por un lado, el vínculo que unía a su familia con la educación y, por otro, la presencia de los medios de comunicación en su hogar.

En 1998, a los 10 años de edad desea ser docente. Al año siguiente, ese deseo comienza a tomar forma desde el interés por el profesorado de Ciencias Políticas. 
Revista de la Escuela de Ciencias de la Educación, año 11, número 10, enero a diciembre de 2015. Páginas 249-268. ISSN 1851-6297. ISSN EN LINEA 2362-3349. HISTORIAS DE VIDA DE ESTUDIANTES UNIVERSITARIOS MIGRANTES. GUADALUPE R. LEIVA

Para continuar con sus estudios secundarios, sus padres deciden inscribirlo en la Escuela Técnica de Montecarlo. Durante varios meses insistió a sus padres para que lo cambien de colegio y finalmente en el mes de junio del 2000, Daniel regresa a la Escuela Normal donde había realizado su educación primaria, ahora en las aulas de la escuela secundaria, hasta finalizar sus estudios.

En el 2003 opta por la orientación de Humanidades y Ciencias Sociales, desde ese año al 2005 recuerda que ejercía como catequista de la parroquia de su barrio.

Durante los primeros días del mes de diciembre del año 2005 viaja a Posadas junto con sus padres a inscribirse en la carrera de profesorado en Ciencias Políticas de un instituto superior privado católico. Días más tarde, luego de conversar con sus padres sobre su futuro profesional decide retirar su legajo de esta institución y busca nuevas ofertas educativas.

Navegando en internet, descubre que en una universidad pública de la región -ubicada en Resistencia, capital de la provincia del Chaco- se dictaba la carrera de Ciencias de la Educación. Luego de la navidad, viaja a Resistencia con su madre a inscribirse en dicha carrera y juntos buscan alojamiento para alquilar para el año siguiente. Ese mismo día, una vez registrada su inscripción en la carrera, regresan a la ciudad de Montecarlo.

En el mes de marzo de 2006, durante el fin de semana previo al ingreso del cursillo a la carrera, se muda a Resistencia acompañado por sus padres, quienes regresan en el mismo día debido a sus responsabilidades laborales, en sus relatos él remarca que este hecho fue el que marco la "caída al vacío".

En el mes de marzo, inician las clases del primer cuatrimestre. Desde el inicio hasta el mes de abril, recuerda que extrañaba a su familia, su lugar y sus amigos; las llamadas por celular y los mensajes de texto le permitían estar en contacto.

Con la llegada de la fecha de conmemoración de la pascua cristiana, Daniel regresa a su ciudad natal, quedándose una semana más ya que durante esa semana en la facultad se llevaban a cabo las mesas de exámenes finales con suspensión de clases.

El último lunes del mes de abril regresa a Resistencia para cursar y rendir el primer parcial. Sin embargo, no se presenta a rendir, avisa a su familia que no va a quedarse y les comunica su deseo de dejar sus estudios y volver a Montecarlo.

Por consejo de su madre, quien aprueba su decisión, cuando decide retirar su legajo de la facultad no puede hacerlo ya que ésta se encontraba cerrada. Frente a dicha situación y con un fin de semana libre, se sienta a estudiar para los siguientes exámenes parciales que los aprueba luego.

Sus deseos de volver a su ciudad natal parecen haberse perdido en el afán de estudiar, poniéndose a prueba en cuanto a su rendimiento académi- 
Revista de la Escuela de Ciencias de la Educación, año 11, número 10, enero a diciembre de 2015. Páginas 249-268. ISSN 1851-6297. ISSN EN LINEA 2362-3349. HISTORIAS DE VIDA DE ESTUDIANTES UNIVERSITARIOS MIGRANTES. GUADALUPE R. LEIVA

co. A la semana, rinde y aprueba dos materias del primer nivel (Psicología e Introducción a las Ciencias Sociales).

Es así, que llegando al mes de junio, espera la nota del último parcial para regularizar las tres primeras materias de la carrera y regresa a Montecarlo a pasar sus vacaciones de invierno. Durante esos días visita a sus amigos, familiares y además estudia para su primer examen final.

Al regresar a Resistencia, aprueba su primera materia (Corrientes del Pensamiento Contemporáneo), terminada la instancia evaluadora sube a un micro que lo lleva de regreso a Montecarlo.

En el mes de agosto de 2006, regresa a Resistencia para continuar los estudios regularmente, sus días transcurren entre las clases y sus horas de estudio. En los momentos en que extrañaba a su familia, salía a caminar y mirar vidrieras con el sólo objetivo de que el tiempo transcurriera para llegar a su casa para dormir.

En su relato, recuerda que el estar ahí le hacía sentir que no estaba preparado para alejarse de su familia, por lo cual a fines de septiembre decide definitivamente dejar la carrera y volverse, emprendiendo una nueva mudanza.

Durante unos meses y estando en su casa, rodeado de su familia, toma ese tiempo para la reflexión acerca de su futuro profesional. En ese momento, su hermana mayor decide inscribirse e iniciar también la carrera de Ciencias de la Educación. Tal es así que Daniel junto a su hermana viajan rumbo a Resistencia a fines de diciembre, para realizar las inscripciones correspondientes y buscar un alquiler para los ambos.

En el año 2007, regresa a la ciudad de Resistencia para continuar con sus estudios universitarios, pero esta vez acompañado por su hermana mayor, que también estudiaría la misma carrera universitaria. Son compañeros en cursado de las materias del segundo cuatrimestre, y con ello, comparten grupo de estudio.

En aquellos tiempos, cuenta Daniel que estudiaba y realizaba los trabajos prácticos con el grupo de compañeros que su hermana había formado cuando inicia la carrera. Durante todo el año lectivo no regresaron excepto en el periodo vacacional de verano.

Durante los meses de vacaciones, su hermana decide no continuar con sus estudios, entonces regresa solo en el mes de febrero para rendir sus exámenes finales. Daniel continúa sus estudios acompañado del mismo grupo de trabajos con el cual había iniciado el año anterior.

Es en el transcurso del 2008 surge en él un interés particular por la carrera de Comunicación Social, intenta inscribirse pero olvida la fecha de inscripción y por lo cual no la inicia durante el año 2009.

Al año, cambia de grupo de estudio debido al régimen de correlatividades para realizar el cursado de las materias y conforma un nuevo grupo y de estudio. Viaja a Montecarlo para festejar las fiestas y se queda por el periodo vacacional. 
Revista de la Escuela de Ciencias de la Educación, año 11, número 10, enero a diciembre de 2015. Páginas 249-268. ISSN 1851-6297. ISSN EN LINEA 2362-3349. HISTORIAS DE VIDA DE ESTUDIANTES UNIVERSITARIOS MIGRANTES. GUADALUPE R. LEIVA

Luego del período de vacaciones 2007-2008, a partir de haber aprobado una materia en el mes de febrero, comienza a plantearse la importancia de focalizar en el estudio para lograr su meta personal y profesional.

A fines del mes de septiembre realiza sus prácticas de la enseñanza. Y los primeros días del mes de noviembre su padre se enferma y es trasladado a la capital de la provincia de Corrientes para realizar su tratamiento, el día 10 del mismo mes su padre fallece y en compañía de su madre retorna a Montecarlo acompañando el féretro de su padre.

Solo se queda en su ciudad natal un día, a fin de acompañar a su familia en el duelo; entiende que cada uno debe seguir con sus obligaciones y regresa a la ciudad de Resistencia a continuar con sus estudios. Su madre viaja a Resistencia los primeros días del mes de diciembre y lo ayuda en su mudanza.

El 23 de diciembre, como lo habría realizado desde el año 2008, viaja a Montecarlo y llega a su el día 24 para celebrar la navidad. Es en este viaje, que le comenta a su madre, su interés por la Carrera de Comunicación Social y sus deseos de inscribirse y ella, por su parte, lo apoya en la decisión. Coincidentemente, su hermano menor abandona la carrera de Ingeniería Forestal iniciada en Misiones y planea estudiar Medicina en la ciudad de Corrientes.

En el año 2011, vuelve a Resistencia para estudiar los finales que adeudaba, durante el segundo cuatrimestre del profesorado en Ciencias de la Educación e inicia el cursado de la licenciatura en Ciencias de la Educación. En el mes de diciembre del mismo año, llega su mamá desde Montecarlo para acompañarlo en su último examen. Y el 22 del mismo mes, rinde Historia de la Educación y por la tarde Organización Educativa y se recibe de profesor.

Al día siguiente, regresa a Montecarlo. Al finalizar las fiestas navideñas, con su hermano, deciden volverse pero esta vez, para realizar sus correspondientes inscripciones en carreras de grado en la el campus universitario de la ciudad de Corrientes. Al finalizar los trámites regresan a Misiones, pero solo por unos días.

El 1 de enero de 2012, Daniel y su hermano llegan a la ciudad de Corrientes para instalarse y ambos inician el cursado regular del primer año durante el mes de marzo; Daniel inicia la carrera de Comunicación Social, y su hermano por su parte, comienza Medicina.

- Caso 2: Historia de vida de Jimena (14)

El 2 de Noviembre de 1989 en la ciudad de Esperanza (Santa Fe) nace Jimena, siendo la segunda hija del matrimonio de Amanda y Rubén, ambos oriundos de la provincia de Entre Ríos. Sus primeros años y la primera etapa de su infancia transcurren en dicha localidad.

Durante los años 1993 y 1994, inicia el jardín de infantes y pre escolar en un colegio privado católico. Sus relatos sobre su infancia están muy ligados a la distribución de tiempo y actividades que realizaba por aquellos días, re- 
Revista de la Escuela de Ciencias de la Educación, año 11, número 10, enero a diciembre de 2015. Páginas 249-268. ISSN 1851-6297. ISSN EN LINEA 2362-3349. HISTORIAS DE VIDA DE ESTUDIANTES UNIVERSITARIOS MIGRANTES. GUADALUPE R. LEIVA

cuerda que dormían hasta el medio día y de ahí iba junto con sus hermanos rumbo al colegio en un colectivo escolar que los pasaba a buscar. Mencionó a su chofer del transporte escolar: el señor Gandini y a la esposa de éste. Ella, viajaba vestida con un guardapolvo blanco y era la responsable de cuidarlos en el recorrido desde su casa a la escuela y viceversa.

Al año siguiente, inicia el primer grado de la escuela primaria en el mismo colegio. A fines de este año, la operan de las amígdalas.

Durante los primeros meses del año 1996, por razones laborales de su padre, migran a la localidad de Las Toscas a $417 \mathrm{~km}$ de la capital de la provincia de origen, al norte de la provincia litoraleña.

En relación a su primer movimiento migratorio, Jimena comenta que sus compañeritos de la escuela le decían que se irían a vivir al fin del mundo respecto del lugar al cual irían a con su familia. Ella recuerda que por ese entonces, junto a sus hermanos emprendieron una feria de garaje y en este emprendimiento, ella vende sus juguetes; luego, al regresar sus padres, les asignan un castigo por tal acción.

De ese primer año desde que se mudaron a Las Toscas, recuerda que la única actividad que realizaban junto con sus dos hermanos era ir a la escuela y jugar entre ellos en su casa. En sus relatos, no menciona ningún amigo ni vinculo establecido con el exterior durante ese periodo, exceptuando la escuela, pero de la que tampoco se explaya mencionando hechos sobre este año.

Al año siguiente, luego de haber visto al grupo de Boys Scout en un acto cívico en la plaza, le pide a su padre que la inscriba en el grupo; así es que ella comienza a realizar actividades extra escolares en este grupo a partir de 1997 y al tiempo, se suman sus hermanos y su padre que luego ejerce como guía de grupo Scout.

Otra de las actividades que en sus relatos recupera en relación a estos años es, que en paralelo, forma parte del grupo Infancia Misionera, la cual es una obra misional pontificia para la animación misionera de los niños y de sus educadores.

Sus relatos la llevan a recordar que en el año 2001 inicia el cursado del EGB 3, se detiene a comentar sobre un hecho en particular que hace referencia al interés de su madre para que curse su educación secundaria en la ciudad de Reconquista (ubicada a $103 \mathrm{~km}$ de Las Toscas), ante lo cual ella junto con su hermana menor se oponen ya que no querían emprender otro viaje. En el año 2003, elige el bachillerato con orientación docente en Las Toscas.

Durante los años de cursado del Polimodal ella menciona la realización de múltiples actividades extra-curriculares: la participación en la estudiantina, la confección de carrozas, el centro de estudiantes (relata que en ese momento, ella fue electa como Presidente del mismo), los trabajos en el diario local "EI Buen amigo" y en el programa televisivo de Las Toscas, donde comentaban sobre las actividades que realizaban en la escuela secundaria. 
Revista de la Escuela de Ciencias de la Educación, año 11, número 10, enero a diciembre de 2015. Páginas 249-268. ISSN 1851-6297. ISSN EN LINEA 2362-3349. HISTORIAS DE VIDA DE ESTUDIANTES UNIVERSITARIOS MIGRANTES. GUADALUPE R. LEIVA

Su primera elección profesional, es la de ser maestra jardinera en el año 2005; pero frente al comentario de su madre sobre su destreza en relación a las manualidades, deserta de la idea. Un año después, viendo un programa de televisión, se interesa por quien está participando en una sección televisiva, consulta sobre la profesión de la misma, al tener respuesta de que ésta era cientista de la educación empieza a averiguar sobre la carrera. Decide a fines de ese año que quiere estudiar Ciencias de la Educación en Resistencia.

Una vez terminada la escuela secundaria, se muda a la ciudad de Resistencia e inicia sus estudios universitarios en Ciencias de la Educación. A fines de ese año, conoce a su compañera de estudios, como también, a un vecino de su pensión que es oriundo de Las Toscas y que inicio sus estudios universitarios en Resistencia unos años atrás.

Ya en año 2010, cursando el cuarto año de carrera, ella empieza a buscar trabajo junto con su compañera de estudio. A fines de este año la llaman para realizar una entrevista de trabajo para un puesto laboral en el sistema de plataformas educativas virtuales de la universidad, dependiente de Rectorado y ubicado en la ciudad capital de la provincia de Corrientes.

En marzo del año 2011, se inscribe a cursar las materias del 5to año de la carrera y la llaman para trabajar donde había realizado la entrevista a fines del año 2010, tal es así que empieza a trabajar en Corrientes a escondidas de sus padres. De lunes a viernes dividía su jornada entre el estudio y el trabajo, para lo cual, cruzaba todos los días el puente "General Manuel Belgrano" que une ambas ciudades. En el mes de diciembre se recibe de profesora en Ciencias de la Educación.

El último movimiento migratorio que emprende, se realiza en el año 2012, cuando se muda a la ciudad de Corrientes y continúa con su trabajo iniciado durante el año 2011.

En sus relatos, hace mención a los acontecimientos que fueron suscitándose en torno a sus distintos movimientos migratorios, y estos están relacionados particularmente con los lugares en donde fue asentándose, primero con su familia y luego sola.

\section{Trabajo con las herramientas de objetivación de la información}

El trabajo con las herramientas de objetivación para cada uno de los casos ha permitido la visualización, a modo gráfico, de los desplazamientos de tipo geográfico emprendidos por cada uno de los recientes graduados. En ambos gráficos la línea cronológica de los hechos narrados se identifica la relación entre espacio, tiempo y significación otorgada para cada momento, según las voces de los entrevistados. A continuación, se presentan en dos gráficos (Gráfico 1: Caso 1 "Daniel” y Gráfico 2: Caso 2 “Jimena"). 
Revista de la Escuela de Ciencias de la Educación, año 11, número 10, enero a diCIembre de 2015. PÁginas 249-268. ISSN 1851-6297. ISSN EN LÍNEA 2362-3349. HISTORIAS DE VIDA DE ESTUDIANTES UNIVERSITARIOS MIGRANTES. GUADALUPE R. LEIVA
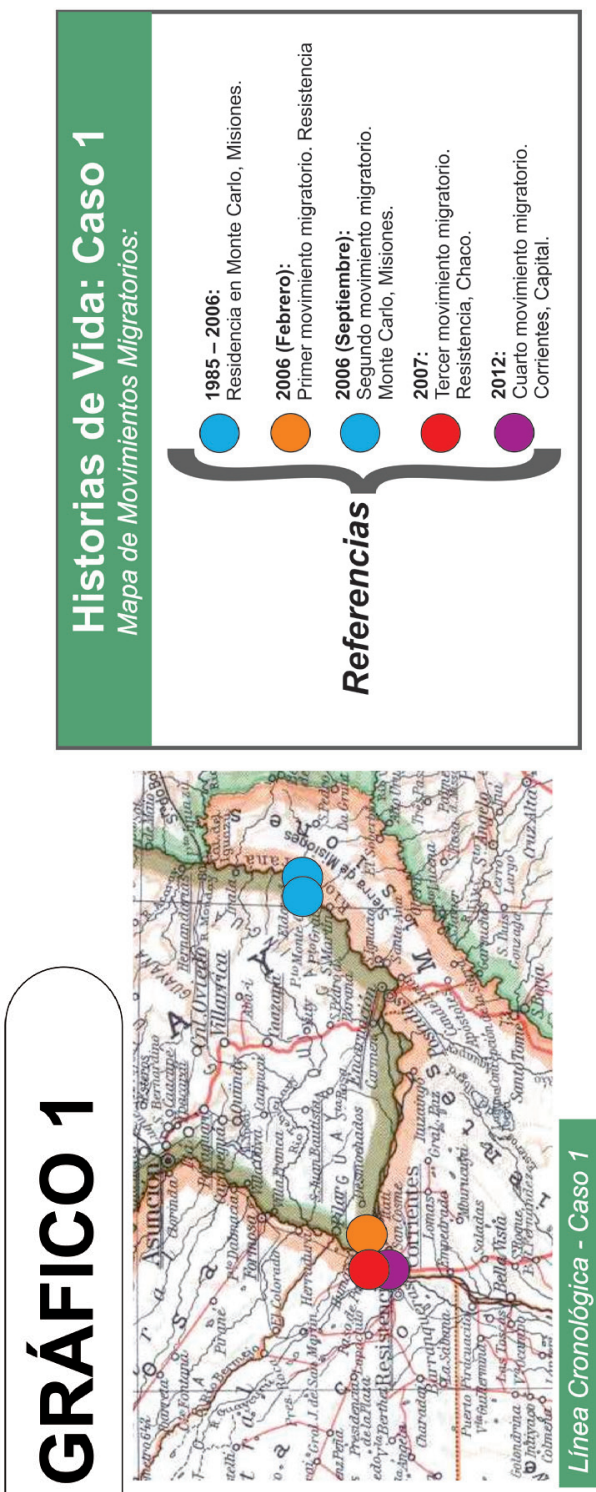

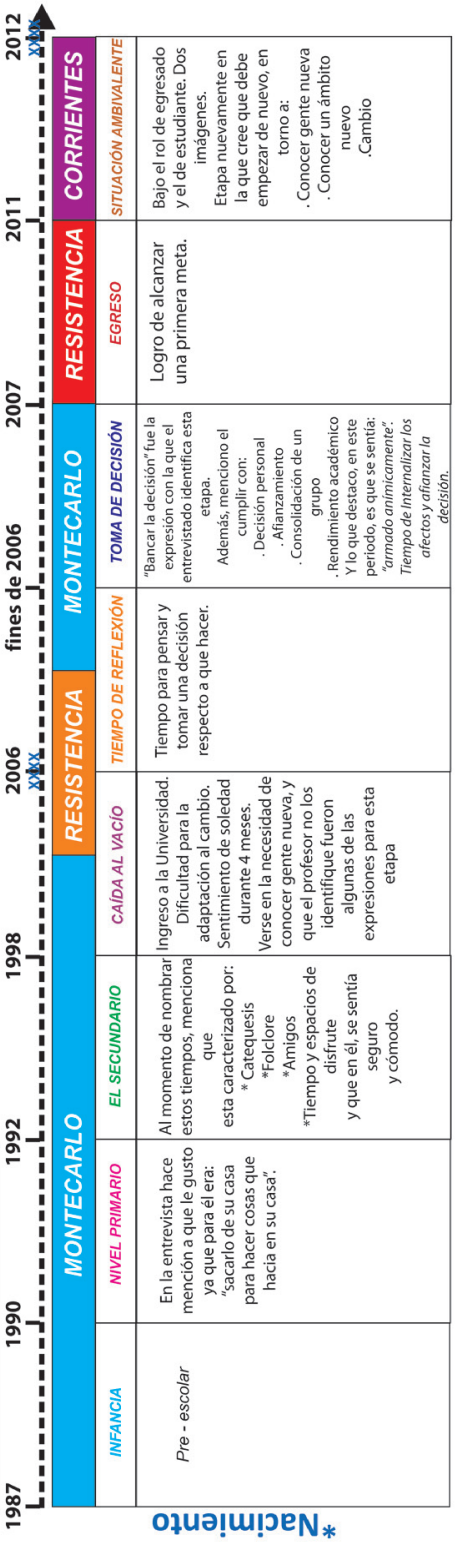


Revista de la Escuela de Ciencias de la Educación, año 11, número 10, enero a diciembre de 2015. PÁginas 249-268. ISSN 1851-6297. ISSN EN LINEA 2362-3349. HISTORIAS DE VIDA DE ESTUDIANTES UNIVERSITARIOS MIGRANTES. GUADALUPE R. LEIVA

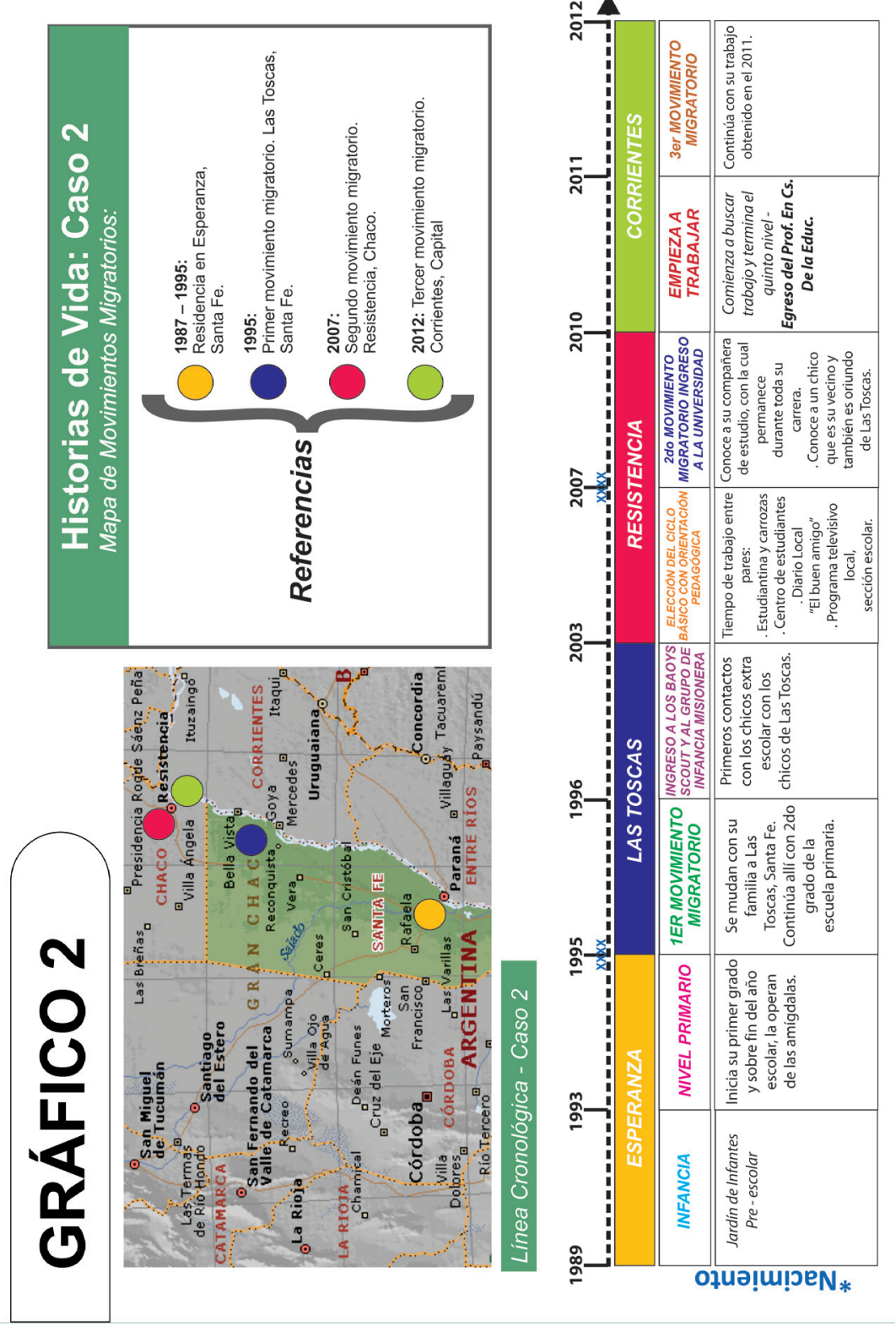


Revista de la Escuela de Ciencias de la Educación, año 11, número 10, enero a diciembre de 2015. Páginas 249-268. ISSN 1851-6297. ISSN EN LINEA 2362-3349. HISTORIAS DE VIDA DE ESTUDIANTES UNIVERSITARIOS MIGRANTES. GUADALUPE R. LEIVA

\section{Primeros avances en la elaboración de hipótesis interpretativas}

Los primeros avances sugieren que los sujetos que migran se ven despojados de sus costumbres, rutinas, vínculos, representaciones, relaciones sociales, y por lo tanto se ve afectada su identidad construida para con el lugar de pertenencia. Los movimientos migratorios, rompen con las configuraciones vinculares de los sujetos; por lo cual, la migración es vivida como ruptura, separación y desarraigo.

Tal como ha sido visualizado en el Caso 1: HdV de Daniel (Gráfico 1), el salir de la ciudad natal rumbo a una ciudad en la que no sólo no conocía geográficamente, sino en la que además se encontraría sólo, puede ser pensado en términos de desarraigo, colocándolo en una situación de no pertenencia de un "no lugar" (15), de incertidumbre. Los contextos significativos, tanto la familia, los amigos y el entorno afectivo son modificados, produciéndose alteraciones y esto se convierte en una amenaza para la realidad del sujeto, por lo que experimenta y sufre rupturas en su biografía subjetiva.

Tal como lo describen Grinberg y Grinberg (1984, pp. 25-26), la vida implica necesariamente, pasar por una sucesión de duelos; la evolución da tiempo al yo, a la elaboración de las pérdidas y al restablecimiento de los momentos transitorios de trastorno de la identidad. Sintiéndose en un lugar desconocido y ajeno, los sujetos que emigran mediante la interacción que construyen con el medio y con los otros individuos, logra encontrar sus apoyaturas (16) en este proceso de reconstrucción de su propia identidad.

Pensar en los movimientos migratorios, nos lleva a plantearnos acerca de los diferentes tipos de ansiedades que los sujetos experimentan frente a los cambios originados por el fenómeno de la migración, tal como lo menciona el sujeto Caso 1:

Yo era una pelota de emociones, de emociones como cruzadas era una cosa de extrañar, me gustaba la carrera pero extrañaba demasiado o aunque sea quería saber cómo estaba mi familia, quería verlos, como que no estaba maduro yo saqué esa conclusión yo no estaba lo suficientemente maduro, a nivel académico me iba perfecto pero a nivel emocional no. (HdV 1: Daniel - Entrevista 1) (17).

En la experiencia de migración del caso de Daniel, puede verse que el momento que denomina "caída al vacío" en la primera entrevista, se constituye en un momento crucial para el sujeto, convirtiéndose en una intensa experiencia de desposesión, de despojamiento y pérdida que moviliza las energías y los mecanismos del trabajo del duelo en términos de Kaës (1997, p.34).

Para Daniel el hecho de dejar su ciudad natal, las reuniones familiares y los días de encuentro con sus amigos, para pasar a ir a vivir a un lugar desconocido, donde además no tenía con quien compartir su día a día, fue vivido 
Revista de la Escuela de Ciencias de la Educación, año 11, número 10, enero a diciembre de 2015. Páginas 249-268. ISSN 1851-6297. ISSN EN LINEA 2362-3349. HISTORIAS DE VIDA DE ESTUDIANTES UNIVERSITARIOS MIGRANTES. GUADALUPE R. LEIVA

por éste como una separación y un desgarramiento, quedando evidenciado, el día que sus padres lo acompañan a la ciudad donde reside la universidad y lo dejan instalado ese mismo día. Este hecho, es vivido como punto central de la ruptura en la regularidad de la vida interna del sujeto.

Esto, puede evidenciarse en la segunda entrevista cuando el sujeto denomina "caída al vacío" al primer movimiento migratorio realizado:

Para mí fue una caída libre, porque nunca me voy a olvidar cuando me voy a despedir a mis padres, porque ahí me di cuenta que me iba a quedar solo y por meses y después y conocer y ahora me está pasando lo mismo en la otra carrera. Gente nueva poco saben que yo ya soy profesor porque yo no quiero que haya cierto prejuicio en ellos además soy tímido y me cuesta vincularme al principio, entonces yo sabía que esa caída libre me sirvió y yo sabía que me iba a ser difícil encontrar un grupo, conocer un grupo. Y acá tenía que comenzar de cero y después la profesora al principio no te identifica, y te habla como si fuera uno más... eso y lo mismo con los compañeros y si mi objetivo es estudiar, yo vengo a estudiar. (HdV 1: Daniel - Entrevista 2) (18).

Los sujetos que migran se ven necesitados de un espacio potencial que les sirva de lugar de transición, permitiéndole a su vez, organizar sus partes del yo disueltas. Los momentos de crisis son superados y elaborados, mediante la transicionalidad como la elaboración de una experiencia de la ruptura.

En este sentido, Kaës recupera los aportes de Winnicott, entendiendo la transicionalidad como la "zona intermedia de experiencia y este proceso de pasaje (de transición) entre dos estados subjetivos: la transicionalidad es la disposición de una experiencia de ruptura en la continuidad" (1997, p. 61); mediante la cual, los sujetos tendrán la posibilidad de un verdadero crecimiento de la personalidad.

Para Daniel, su tercer movimiento migratorio a la ciudad de Resistencia acompañado por su hermana, la que podemos afirmar, se convierte en el sostén en esta nueva etapa de su vida en que decide retomar sus estudios; esto le permite a Daniel la posibilidad de la conformación de la transicionalidad para la reelaboraciones sucesivas de la propia identidad.

Por su parte, el grupo de estudios con el cual Daniel continúa una vez que su hermana mayor abandona los estudios puede ser considerado como objeto transicional. Daniel hace mención al grupo de trabajo con quien su hermana compartía y con quienes después de la partida de su hermana continúa trabajando:

En este grupo que también pertenecía a mi hermana era un grupo que parece que nos conocíamos de toda la vida, eh... se trabajaba 
Revista de la Escuela de Ciencias de la Educación, año 11, número 10, enero a diciembre de 2015. Páginas 249-268. ISSN 1851-6297. ISSN EN LINEA 2362-3349. HISTORIAS DE VIDA DE ESTUDIANTES UNIVERSITARIOS MIGRANTES. GUADALUPE R. LEIVA

bien, se estudiaba perfectamente bien y nos entendíamos también nos complementábamos en el momento de recreación, tomábamos mates, nos reíamos, mirábamos programas y nos reíamos o sea nos complementábamos bien, para mí eso es un grupo, es decir yo al sentirme cómodo con eso al sentirme tanto en lo académico como a nivel personal digamos. (HdV 1: Daniel - Entrevista 2).

La identificación como parte de la construcción de la identidad se produce junto con la diferenciación que se establece mediante los intercambios dentro de las relaciones sociales, tal como lo visualizamos en la historia de vida de Daniel.

Cada movimiento migratorio se ha inscripto de un modo particular en la historia personal del Caso 2: HdV de Jimena. El sujeto narra diversas migraciones (Gráfico 2), la primera es vivenciada en la infancia, a la fecha, ha realizado tres migraciones, la segunda la realizó al momento de inicio de la carrera y la tercera posterior a su egreso.

En los movimientos migratorios durante la niñez pueden pensarse algunos aspectos que podrían ser positivos frente al cambio, ya que puede convertirse en una situación menos traumática debido a que el entorno del niño es reducido y por lo general, migran con los padres y/o hermanos. Grinberg y Grinberg (1984) en cuanto a esto, plantean que los modos en los que serán asimiladas las experiencias migratorias pueden guardar relación con la edad en la que se produce el movimiento:

El traslado se realiza como acompañado de una capa protectora, de una envoltura que lo contiene. Por otra parte, es más hábil para imitar, para dejarse impregnar por impresiones nuevas, está más abierto al aprendizaje y por lo tanto, más capaz de asimilar un nuevo lenguaje, costumbres, etc. (1984, pp. 137-138)

Los autores también remarcan que los niños, al no comprender los motivos que provocan la migración y al no poder tomar decisiones respecto de si quieren ir o no, vivencia la migración de un modo particular ya que el la experiencia migratoria se experimenta en un entorno inmediato del niño que lo ha "sacudido", término acuñado por Grinberg y Grinberg (1984, p.138).

Jimena en la primera entrevista relataba el modo en el que ella con solo seis años percibía junto con sus hermanos el despojo que iban a pasar debido a su primera migración:

Y yo me acuerdo que cuando me vine a vivir a Las Toscas mis vecinitos me decía, en Las Toscas se termina el mundo, en Las Toscas son todos aborígenes, no hay más nada después de ahí... y agarré, con mis hermanos agarramos y pusimos todas nuestras cosas en el tapial de nuestra casa y vendíamos nuestros juguetes y mi mamá nos re puteo después, porque no se qué se nos ocurrió a nosotros 
Revista de la Escuela de Ciencias de la Educación, año 11, número 10, enero a diciembre de 2015. Páginas 249-268. ISSN 1851-6297. ISSN EN LINEA 2362-3349. HISTORIAS DE VIDA DE ESTUDIANTES UNIVERSITARIOS MIGRANTES. GUADALUPE R. LEIVA

que teníamos que hacer una feria y vender nuestros juguetes. Como desprendiéndonos de nuestras pertenencias. (HdV 2: Jimena - Entrevista 1) (19).

El desprenderse de sus juguetes, le significó a Jimena desprenderse de las representaciones internas que esos objetos tenían para ellos en relación a un momento dado y al sentido de pertenencia que habían creado para con el lugar que dejarían.

El despojo vivenciado en las experiencias de movimientos migratorios, pueden promover hacia el interior del grupo familiar, un apego de cada uno de los miembros con su familia; esta vuelta simbólica a la propia familia puede ser considerada como el correlato de la pérdida de un lugar social estable y reasegurador; es por ello que los miembros de una familia sobrecargan los vínculos hacia el interior del grupo familiar, refugiándose en ellos que representan lo seguro y lo permanente.

Jimena recuerda que durante el primer año que se asentaron en el lugar de su primer movimiento migratorio, más allá de ir a la escuela no hacían otro tipo de actividad, y que todo estaba centrado hacia el interior de su casa:

Cuando llegamos a Las Toscas no hablábamos con nadie, íbamos a la escuela y de ahí a nuestra casa, estábamos los tres solos en casa, leyendo y jugando entre nosotros. (HdV 2: Jimena - Entrevista 2) (20).

Es por ello, que luego del ver a los Boys Scout en un desfile cívico militar en la plaza central, ella decide que quiere formar parte del grupo:

Yo fui la primera en mi casa que intento salir de mi casa, o sea, fui la primera de los tres que busco algo para hacer en Las Toscas cuando vinimos para allá (...). Eso creo que fue como algo importante en mi vida porque fue mi primera relación con alguien que no fuéramos nosotros tres desde que estábamos ahí. (HdV 2: Jimena - Entrevista 2).

El recurrir a nuevos grupos y vínculos, los sujetos que realizan movimientos migratorios constituyen un modo transicional de sostén provisorio en pos de una elaboración de la migración.

Jimena en su relato dio cuenta acerca de no sentirse perteneciente a ningún lugar geográfico en particular:

Lo primero que se me ocurre al pensar en mi vida, hubo un tiempo que viví en Esperanza, otro que viví en Las Toscas, otro en Resistencia y el último hasta ahora en Corrientes. Que creo que tiene que ver con el que nunca me sentí de ningún lugar. Tuve una época en cada lugar, momentos en lugares distintos, y si me preguntan 
Revista de la Escuela de Ciencias de la Educación, año 11, número 10, enero a diciembre de 2015. Páginas 249-268. ISSN 1851-6297. ISSN EN LINEA 2362-3349. HISTORIAS DE VIDA DE ESTUDIANTES UNIVERSITARIOS MIGRANTES. GUADALUPE R. LEIVA

de dónde soy, titubeo y digo que soy de Entre Ríos, porque no me siento de ningún lugar. Desde que nací a los 7 años viví en el mismo lugar, de los 7 a los 18 en Las Toscas, de los 18 a los 22 en Resistencia, y ahora ya en Corrientes. (HdV 2: Jimena - Entrevista 2)

\section{Primeras reflexiones a propósito de los avances y resultados}

Esta investigación estudio los movimientos migratorios de tipo geográficos que realizan los estudiantes universitarios de grado en la región NEA de la Republica Argentina y analiza los posibles impactos en el trayecto de formación.

En este sentido, se considera que la situación vivida a partir del movimiento migratorio es experimentada como una ruptura y es la huella enunciada como acontecimiento por cada uno de los entrevistados la que se recupero mediante la reconstrucción de las historias de vida que aquí se presentaron.

La elaboración de las experiencias de la ruptura, vividas a partir de la migración, se constituye en la experiencia inaugural para re elaboración de nuevos vínculos y constitutiva de la propia identidad de los sujetos. Las experiencias de múltiples migraciones que no logran ser elaboradas a lo largo de la vida, ponen en riesgo no sólo el sentido de pertenencia, sino también, la identidad de los sujetos.

Se ha visualizado así también, que es posible que aquellos estudiantes universitarios que han debido realizar uno o varios desplazamientos de tipo geográficos al momento de iniciar sus estudios en la universidad, ha puesto al sujeto a prueba en relación a las configuraciones vinculares frente a lo imprevisto, lo incierto, lo ajeno del otro y la capacidad de transformar las situaciones críticas en experiencias de aprendizaje mediante la interacción que construyen con el medio y con los otros individuos, de este modo, los sujetos logran restablecer la estabilidad a su self y a su sentimiento de identidad.

La universidad es entendida como ese espacio transicional que propicia la sutura, a partir las uniones creativas que emergen de la trama de las relaciones intersubjetivas y que son expresadas mediante el sentido de pertenencia; en el caso de los estudiantes migrantes, pertenencia a un grupo, a una institución.

\section{Notas bibliográficas}

(1) Dirigida por la Mgter. María Cristina Alonso. U.N.N.E.

(2) Led by the Magister. María Cristina Alonso. U.N.N.E.

(3) Beca de investigación dirigida por la Mgter. M. Cristina Alonso. Res. 1015/12 C. S. de la Secretaria General de Ciencia y Técnica (SGCyT) de la Universidad Nacional del Nordeste (UNNE).

(4) Kaës (1992) es quien reelabora el concepto freudiano de apuntalamiento, que sostiene que el psiquismo en su conformación se apoya en las funciones corporales, a lo que agrega que éste, se apoya también sobre el grupo, la cultura y el propio aparato psíquico; 
Revista de la Escuela de Ciencias de la Educación, año 11, número 10, enero a diciembre de 2015. PÁginas 249-268. ISSN 1851-6297. ISSN EN LINEA 2362-3349. HISTORIAS DE VIDA DE ESTUDIANTES UNIVERSITARIOS MIGRANTES. GUADALUPE R. LEIVA

es mediante está conceptualización, que Kaës plantea el concepto de apoyatura a partir de la concepción grupal del psiquismo (1992, pp.15-35).

(5) "Las representaciones que tienen los alumnos de Ciencias de la Educación sobre la formación y los espacios de acompañamiento" Beca de Iniciación. Directora: Mgter. M. Teresa Alcalá. Co - Directora: Mgter. M. Cristina Alonso. Res. N 1115/09 C. S. (20102013) SGCyT UNNE.

(6) Este término se basa en la huella resultado de una situación vivída como desconcertante y dramática. Es desde la noción fenomenológica, donde la noción de "huella" nos permite pensar a partir de lo abordado por Ricoeur al afirmar que ésta, se "construye sobre la base de ser-afectado por el acontecimiento del que se da testimonio por narración, tras la modificación de las experiencias pasadas en función de las nuevas" (2013, p.93)

(7) El concepto de cambio catastrófico fue introducido en el pensamiento psicoanalítico por Bion denominó cambio catastrófico, al proceso mediante el cual la desintegración y la integración se correspondería con las descripciones de Melanie Klein relativas a los movimientos de la posición esquizoparanoide a la depresiva y viceversa (1981, pp.777-788). Bion afirma entonces, que la oscilación permanente entre los procesos de desintegración e integración, pasan siempre por la turbulencia del cambio catastrófico, lo que se constituiría en la condición inherente al cambio psíquico.

(8) En La poétique de la rêviere, Bachelard presenta la noción de "núcleo de infancia", la cual, está presente en el alma humana y puede expresarse mediante la memoria.

(9) Stake plantea que "el estudio de casos es el estudio de la particularidad y de la complejidad de un caso singular, para llevar a comprender su actividad en circunstancias importantes" (1999, p.12).

(10) Op. Cit. Resolución N 1115/09 y Resolución N 1015/12 C. S. SGCyT UNNE.

(11) Mediante la que por reiterados encuentros cara a cara entre el entrevistador y los informantes la investigación se dirigirá hacia la comprensión de las perspectivas que tienen los informantes respecto de sus vidas, experiencias o situaciones, tal como la expresan con sus propias palabras, tratando de evitar las respuestas cortas.

(12) En la investigación cualitativa, el trabajo sobre historias de vida representan con mayor fidelidad los relatos de los individuos. Se sustenta en el relato que un individuo hace de su vida o de aspectos específicos de ella, de los modos como él interpreta los acontecimientos vividos y define en ellas, las situaciones en las que él ha participado.

(13) El nombre "Daniel" es ficticio y ha sido acordado con el entrevistado 1, a fin de resguardar el anonimato.

(14) El nombre "Jimena" es ficticio y ha sido acordado con la entrevistada 2, a fin de resguardar el anonimato.

(15) Entendiendo la noción de "no lugar" en el sentido que lo plantea Augé (2000) ligada a lo temporal y al tránsito, designando dos realidades: los espacios constituidos con relación a ciertos fines y la relación que los individuos mantienen con esos espacios.

(16) Lidia Fernández en su capítulo "Crisis y dramática del cambio" establece ciertas bases para diferenciar catástrofe y crisis; para esta ultima noción considera lo siguiente: "La crisis dice Kaës (1997) es una experiencia de ruptura en el transcurso de las cosas, vivida como un quiebre imprevisto y masivo de los lazos que unen al sujeto con sus apoyaturas", en Butelman (1996, p.192). En este sentido, para Kaës (1997) la experiencia de crisis está dada por la experiencia de ruptura en la continuidad de las cosas, de sí mismo y del entorno y, por lo tanto, en relación con los apoyos con que cuenta el psiquismo. Kaës plantea que además del apuntalamiento de la pulsión sobre las funciones biofisiológicas corporales y el del objeto de amor sobre la madre, existe un apoyo sobre el self [sol] 
Revista de la Escuela de Ciencias de la Educación, año 11, número 10, enero a diciembre de 2015. Páginas 249-268. ISSN 1851-6297. ISSN EN LÍNEA 2362-3349. HISTORIAS DE VIDA DE ESTUDIANTES UNIVERSITARIOS MIGRANTES. GUADALUPE R. LEIVA

y un apoyo de las funciones psíquicas sobre el grupo y la institución en aquello que mediatizan del orden social y cultural mediante formas y procesos propios (1997, p.14).

(17) Entrevista 1 realizada a Daniel el día 3 de abril de 2012 en la ciudad de Corrientes, capital. Entrevistadora: Guadalupe R. Leiva.

(18) Entrevista 2 realizada a Daniel el día 14 de abril de 2012 en la ciudad de Corrientes, capital. Entrevistadora: Guadalupe R. Leiva.

(19) Entrevista 1 realizada a Jimena el día 20 de abril de 2012 en la ciudad de Corrientes, capital. Entrevistadora: Guadalupe R. Leiva.

(20) Entrevista 2 realizada a Jimena el día 27 de abril de 2012 en la ciudad de Corrientes, capital. Entrevistadora: Guadalupe R. Leiva.

\section{Referencias bibliográficas}

- $\quad$ Ardoino, J. (2005). Complejidad y formación. Pensar la educación desde una mirada epistemológica. Colección Formación de Formadores. Buenos Aires: Novedades Educativas.

- Bion, W. (1981). Cambio Catastrófico. Tomo XXX, Nro. 4. Buenos Aires, Revista de Psicoanálisis de la Asociación Psicoanalítica Argentina. Pp. 777-788.

- Candau, J. (2006). Antropología de la memoria. Buenos Aires: Ediciones Nueva Visión.

- Ferry, G. (1997). Pedagogía de la formación. Colección Formación de Formadores. Buenos Aires: Novedades Educativas.

- $\quad$ Freud, S (2006). Lo siniestro. Ensayos del XCVIII al CXLIV. Tomo 7 (1916-1924). Madrid: Biblioteca Nueva.

- $\quad$ Grinberg, L. y Grinberg, R. (1976). Identidad y Cambio. Barcelona: Editorial Paidos.

- $\quad$ (1984). Psicoanálisis de la migración y del exilio. Madrid: Alianza editorial.

- Kaës, R. y otros. (1997). Crisis, ruptura y superación. Análisis transicional en psicoanálisis individual y grupal. Buenos Aires: Ediciones Cinco.

- Kaës R. (1992). Apuntalamiento múltiple y estructuración del psiquismo. En: Grupos, Instituciones y macro contexto. Buenos Aires, Asociación Argentina de Psicología y Psicoterapia de Grupo.

- Real Academia Española. (2009). Diccionario de la lengua española. Vigésima segunda edición. En: http://www.rae.es/rae.html

- Ricoeur, P. (2005). Sobre la traducción. Colección Espacios del saber. Buenos Aires: Paidós.

- $\quad$ Ricoeur, P. (2013). La memoria, la historia y el olvido. Sección de obras de filosofía. Buenos Aires: Fondo de Cultura Económica. 